\title{
CONTINUITY OF GAUSSIAN PROCESSES
}

\author{
BY \\ M. B. MARCUS AND L. A. SHEPP
}

\begin{abstract}
We give a proof of Fernique's theorem that if $X$ is a stationary Gaussian process and $\sigma^{2}(h)=E(X(h)-X(0))^{2}$ then $X$ has continuous sample paths provided that, for some $\varepsilon>0, \sigma(h) \leqq \psi(h), 0 \leqq h \leqq \varepsilon$, where $\psi$ is any increasing function satisfying

$$
\int_{0}^{\varepsilon} \frac{\psi(h)}{h(\log (1 / h))^{1 / 2}} d h<\infty .
$$

We prove the partial converse that if $\sigma(h) \geqq \psi(h), 0 \leqq h \leqq \varepsilon$ and $\psi$ is any increasing function not satisfying $\left({ }^{*}\right)$ then the paths are not continuous. In particular, if $\sigma$ is monotonic we may take $\psi=\sigma$ and $\left({ }^{*}\right)$ is then necessary and sufficient for sample path continuity. Our proof is based on an important lemma of Slepian.

Finally we show that if $\sigma$ is monotonic and convex in $[0, \varepsilon]$ then $\sigma(h)(\log 1 / h)^{1 / 2} \rightarrow 0$ as $h \rightarrow 0$ iff the paths are incrementally continuous, meaning that for each monotonic bounded sequence $t=t_{1}, t_{2}, \ldots, X\left(t_{n+1}\right)-X\left(t_{n}\right) \rightarrow 0$, w.p.l.
\end{abstract}

1. Introduction. Let $X$ be a zero-mean separable stationary Gaussian process with continuous covariance $\rho(h)=E X(s) X(s+h)$. Xavier Fernique (1964) made considerable progress toward a solution of the well-known problem [6], [3], [1] of finding necessary and sufficient conditions on $\rho$ in order that $X$ have continuous sample paths. Define $\sigma^{2}(h)=E(X(h)-X(0))^{2}=2(\rho(0)-p(h))$. Fernique obtained that if $\sigma(h) \leqq \psi(h), 0 \leqq h \leqq 1$, where $\psi$ is monotonic $\uparrow$ (nondecreasing) and satisfies

$$
\int_{0}^{1} \frac{\psi(h)}{h(\log 1 / h)^{1 / 2}} d h<\infty
$$

then $X$ has continuous paths. He also obtained (under some additional conditions) that if $\psi$ is monotonic $\uparrow$ and (1.1) fails to hold then there exists a discontinuous process $X$ (which he gave as a random lacunary Fourier series) with $\sigma(h) \leqq \psi(h)$, $0 \leqq h \leqq \varepsilon$. Modifying his method of random lacunary Fourier series, we obtain (\$3) using a basic inequality of D. Slepian (1962) that if for some $\varepsilon>0, \sigma(h) \geqq \psi(h)$, $0 \leqq h \leqq \varepsilon$, where $\psi$ is monotonic $\uparrow$ and (1.1) fails to hold then the paths of $X$ are discontinuous. As an immediate consequence we find that if $\sigma$ is monotonic $\uparrow$ then

$$
\int_{0}^{\varepsilon} \frac{\sigma(h)}{h(\log 1 / h)^{1 / 2}} d h<\infty
$$

is the necessary and sufficient condition for $X$ to have continuous paths. In particular if the convariance of $X$ is of Polya's type, the question of whether the paths are continuous is settled completely.

Received by the editors January 10, 1969.

Copyright (C) 1970, American Mathematical Society 
M. Nisio [9] has obtained conditions for continuity of $X$ based on the spectral distribution function $F$. As a corollary to the results of [9], it has been proved [10] that if $F\left(2^{n+1}\right)-F\left(2^{n}\right)=s_{n}$ is eventually decreasing then $\sum\left(s_{n}\right)^{1 / 2}<\infty$ is a necessary and sufficient condition for continuity of $X$. We point out by examples (\$5) that neither' $(1.2)$ nor $\sum\left(s_{n}\right)^{1 / 2}<\infty$ is a nasc for continuity among all covariances.

Finally, we observe that a discontinuous process $X$ may or may not possess the following property: call $X$ incrementally continuous if for each monotonic (either increasing or decreasing) bounded sequence $t=t_{1}, t_{2}, \ldots$

$$
X\left(t_{n+1}\right)-X\left(t_{n}\right) \rightarrow 0 \text { as } n \rightarrow \infty
$$

except for a null set (which may depend on $t$ ) of paths $X$. We show ( $\$ 4$ ) that a Gaussian process $X$ with covariance of Polya's type is incrementally continuous if and only if

$$
\sigma(h)(\log (1 / h))^{1 / 2} \rightarrow 0 \quad \text { as } h \rightarrow 0 .
$$

As an immediate consequence we find that there exists a process $X$ which is incrementally continuous yet not sequentially continuous. (A process $X$ is sequentially continuous if $t_{n} \rightarrow t$ implies $X\left(t_{n}\right) \rightarrow$ limit a.s.; for Gaussian processes sequential continuity is equivalent to sample path continuity.)

We would like to acknowledge the help of $\mathrm{R}$. M. Dudley who provided an enlightening example bearing on $\$ 4$, of $\mathrm{H}$. O. Pollak who found the simple proof of Boas' inequality, and of N. D. Ylvisaker who had suggested the use of Slepian's inequality to one of the authors.

2. Fernique's sufficient condition for continuity of paths. The purpose of this section is to prove the following theorem of Fernique [5]. Alternate proofs can be found in [13]-[16].

THEOREM. Let $X$ be a separable stationary Gaussian process with zero mean and let $\sigma^{2}(h)=E(X(h)-X(0))^{2}$. Let $\psi$ be any nondecreasing majorant of $\sigma$, so that

$$
\begin{aligned}
\sigma(h) \leqq \psi(h), & 0 \leqq h \leqq 1, \\
\psi(h) \uparrow, & 0 \leqq h \leqq 1 .
\end{aligned}
$$

If (1.1) holds, then the paths of $X$ are continuous. Note: it is clear that without loss of generality we could take $\psi$ to be the least nondecreasing majorant of $\sigma$,

$$
\psi(h)=\max [\sigma(u): 0 \leqq u \leqq h] .
$$

Proof. By Belyaev's theorem [1] (see [4] for an alternate proof) discontinuous Gaussian processes are unbounded on every set $S$ dense $\left({ }^{1}\right)$ in some interval with probability one, so we need only prove that for some $M$ and $S$

$$
P\{|X(t)| \leqq M, t \in S\}>0
$$

(1) See Appendix 3. 
where $S$ is dense in some interval. Oddly enough, the choice of the subset $S$ is the crucial part of the proof. We shall choose $S=\left\{k / 2_{p}: 0 \leqq k / 2_{p} \leqq 1\right\}$ where $k$ and $p$ are nonnegative integers and $2_{p}=2^{2^{p}}$, following Fernique [5] (see also [8]). $S$ is sparse enough so that (2.6) (below) holds and yet dense enough so that (2.8) (below) also holds. If $t \in S$, let $k(p, t)$ be the largest integer $k$ for which $k / 2_{p} \leqq t$, so that $k(p, t)=\left[2_{p} t\right]$. Writing $\tau(p, t)=k(p, t) / 2_{p}$ we have

$$
0 \leqq \tau(p+1, t)-\tau(p, t)<1 / 2_{p}
$$

and

$$
X(t)=X(\tau(0, t))+\sum_{p=1}^{\infty}(X(\tau(p+1, t))-X(\tau(p, t))
$$

where the series converges because only finitely many terms are nonzero. To prove the right side of (2.5) bounded in $t$ for $X$ in a set of positive measure we proceed as follows. For $p=0,1,2, \ldots ; k=0,1,2, \ldots, 2_{p}-1 ; q=0,1,2, \ldots, 2_{p}-1$, define

$$
\zeta(p, k, q)=X\left(k / 2_{p}+q / 2_{p+1}\right)-X\left(k / 2_{p}\right) .
$$

Let $D^{2}(\zeta)=E \zeta^{2}$ and set $c(p)=b 2^{p / 2}, p=0,1,2, \ldots$, where $b$ will be chosen later. Let $A$ be the event that for some $(p, k, q)$ in the range above, $|\zeta(p, k, q)|>$ $c(p) D(\zeta(p, k, q))$. Thus we have

$$
P(A) \leqq \sum_{p=0}^{\infty} \sum_{k=0}^{2_{p}-1} \sum_{q=0}^{2_{p}-1} P\{|\eta|>c(p)\},
$$

where $\eta=\zeta \mid D(\zeta)$ is standard normal. Since $P\{|\eta|>c\} \leqq \exp \left(-c^{2} / 2\right)$ for $c>1$ we find choosing $b$ large enough that

$$
P(A) \leqq \sum_{p=0}^{\infty}\left(2_{p}\right)^{2} \exp \left(-b^{2} 2^{p-1}\right)<1 .
$$

Thus comp $(A)$ has positive measure and if $X \in \operatorname{comp}(A)$ we have

$$
|X(\tau(p+1, t))-X(\tau(p, t))| \leqq c(p) D(\zeta),
$$

where $\zeta=\zeta(p, k, q)$ and $k=k(p, t), q=(\tau(p+1, t)-\tau(p, t)) 2_{p+1}$. We have

$$
\begin{aligned}
D^{2}(\zeta) & =D^{2}(\zeta(p, k, q))=\sigma^{2}\left(q / 2_{p+1}\right)=\sigma^{2}(\tau(p+1, t)-\tau(p, t)) \\
& \leqq \psi^{2}(\tau(p+1, t)-\tau(p, t)) \leqq \psi^{2}\left(1 / 2_{p}\right)
\end{aligned}
$$

by (2.1), (2.2), and (2.4). Thus from (2.7) and (2.5)

$$
|X(t)| \leqq|X(\tau(0, t))|+\sum_{p=1}^{\infty} c(p) \psi\left(1 / 2_{p}\right)
$$

Again by monotonicity of $\psi$ we have

$$
2^{p / 2} \psi\left(1 / 2_{p}\right) \leqq \int_{2^{p-1}}^{2^{p}} \psi\left(2^{-u}\right) \frac{d u}{\sqrt{ } u}
$$


and so by (1.1) the series in (2.8) converges:

$$
\sum_{p=1}^{\infty} c(p) \psi\left(1 / 2_{p}\right) \leqq b \int_{1}^{\infty} \psi\left(2^{-u}\right) \frac{d u}{\sqrt{ } u}<\infty .
$$

Finally, since $\tau(0, t)$ has only three possible values, it follows directly from (2.8) and (2.9) that (2.3) holds for some value of $M$.

\section{A sufficient condition for the paths to be discontinuous.}

THEOREM. Let $X$ be a stationary Gaussian process with zero mean and let $\sigma^{2}(h)$ $=E(X(t+h)-X(t))^{2}$. Let $\psi$ be any nondecreasing local minorant of $\sigma$, that is for some $\varepsilon>0$

$$
\begin{aligned}
\sigma(h) \geqq \psi(h) \geqq 0, & 0 \leqq h \leqq \varepsilon, \\
\psi(h) \uparrow, & 0 \leqq h \leqq \varepsilon .
\end{aligned}
$$

If (1.1) does not hold for $\psi$ then the paths of $X$ are not continuous.

Proof. We will show that if the hypothesis holds for $\sigma$ there is a separable, zero mean, stationary Gaussian process $Y$ for which, in the range $s \in I, t \in I, I=[0, \varepsilon]$

$$
\begin{aligned}
& E(Y(t)-Y(s))^{2} \leqq K \sigma^{2}(|t-s|), \\
& P(\sup [Y(t): t \in I]=\infty)=1
\end{aligned}
$$

where $K$ is some constant. In other words we will obtain an unbounded process $Y$ whose incremental variance is bounded by a constant times the incremental variance of $X$. Given such a $Y$ the following inequality of Slepian shows that the paths of $X$ are also unbounded and hence discontinuous.

Lemma (SlePiAn). Let $X$ and $Z$ be separable zero mean Gaussian processes such that $E X^{2}(t)=E Z^{2}(t), E X(s) X(t) \leqq E Z(s) Z(t)$ for $s, t \in I$. Then

$$
P(\sup [X(t): t \in I] \geqq M) \geqq P(\sup [Z(t): t \in I] \geqq M) .
$$

We include Slepian's proof for completeness, but defer it to the appendix.

To see that $X$ is discontinuous if $Y$ exists satisfying (3.3) and (3.4), take $Z(t)$ $=(a \eta+Y(t)) / b$, where $\eta$ is a standard normal variable independent of $Y$, and $a$ and $b$ are constants to be chosen. If $b$ is large enough so that $E Y^{2}(t)<b^{2} E X(t)^{2}$ and $K \leqq b^{2}$ we may choose $a$ so that $E X^{2}(t)=E Z^{2}(t)$. We then have $E(Z(t)-Z(s))^{2}$ $=E(Y(t)-Y(s))^{2} / b^{2} \leqq \sigma^{2}(|t-s|)$ and the hypothesis of Slepian's lemma is satisfied. Since the right side of (3.5) is unity for every $M, X$ is unbounded also.

To produce a process $Y$ satisfying (3.3) and (3.4) we take $Y$ to be a random lacunary Fourier series, the stationary Gaussian process defined by

$$
Y(t)=\sum_{n=0}^{\infty} a_{n}\left(\eta_{n} \cos 2^{n} t+\eta_{n}^{\prime} \sin 2^{n} t\right)
$$


where $\left\{\eta_{n}\right\}$ and $\left\{\eta_{n}^{\prime}\right\}$ are independent standard normal sequences and $\sum a_{n}^{2}<\infty$. Note that

$$
\begin{gathered}
E Y(s) Y(t)=\sum_{n=0}^{\infty} a_{n}^{2} \cos 2^{n}(t-s), \\
E(Y(t)-Y(s))^{2}=2 \sum_{n=0}^{\infty} a_{n}^{2}\left(1-\cos 2^{n}(t-s)\right) .
\end{gathered}
$$

We now use the following theorem of Szidon $[12, \S 6.4]$ to show that $Y(t)$ satisfies (3.4) if

$$
\sum\left|a_{n}\right|=\infty
$$

Lemma (SzIDON). If $\sum\left(b_{n}^{2}+c_{n}^{2}\right)<\infty, \sum\left(\left|b_{n}\right|+\left|c_{n}\right|\right)=\infty$, and $\theta_{n+1} / \theta_{n}>\lambda>1$ then the $L^{2}$ function with Fourier series $\sum\left(b_{n} \cos \theta_{n} t+c_{n} \sin \theta_{n} t\right)$ is unbounded.

To show that (3.4) holds if (3.9) holds let $b_{n}=a_{n} \eta_{n}, c_{n}=a_{n} \eta_{n}^{\prime}$ and $\theta_{n}=2^{n}$ so that the series of the lemma is $Y$. Note that $E \sum\left(b_{n}^{2}+c_{n}^{2}\right)=2 \sum a_{n}^{2}$ and so $\sum\left(b_{n}^{2}+c_{n}^{2}\right)<\infty$ a.s. Similarly, $E\left[\exp \left(-\sum\left(\left|b_{n}\right|+\left|c_{n}\right|\right)\right]=\prod\left(E \exp -\left|b_{n}\right|\right) E\left(\exp \left(-\left|c_{n}\right|\right)\right)=0\right.$ by (3.9) and so $\sum\left(\left|b_{n}\right|+\left|c_{n}\right|\right)=\infty$ a.s. It follows from the lemma that $Y(t)$ is a.s. unbounded on $[0,2 \pi]$ and hence [1] on every interval $[0, \varepsilon]$. Thus the problem reduces to finding an $l^{2}$ sequence $a_{n}$ satisfying (3.9) for which the function $Y$ defined by (3.6) satisfies (3.3).

We shall assume first that $\sigma^{2}$ is monotonically increasing and that $\sigma^{2}\left(2^{-n}\right)$ $\leqq 2 \sigma^{2}\left(2^{-n-1}\right), n=1,2, \ldots$, (call this assumption $\mathrm{A}$ ) because if $\mathrm{A}$ holds (which it does if the covariance is of Polya-type, $\sigma^{2}$ then being monotonic and convex) the construction of the $a_{n}$ sequence is simpler. Later we give a construction which works in general. Define $f_{n}^{2}=\sigma^{2}\left(2^{-n}\right)$ and observe that $f_{n}^{2}$ eventually decreases. Define $g_{n}^{2}$ as the largest convex minorant of $f_{n}^{2}$, so that $g_{n}^{2}=f_{n}^{2}$ for certain values of $n$ and between these values, $g_{n}^{2}$ is linear and lies strictly below $f_{n}^{2}$. We claim first that $g_{n+1}^{2} \leqq g_{n}^{2} \leqq 2 g_{n+1}^{2}$. The first inequality holds because $g_{n}^{2}$ is convex and tends to zero. To prove the second inequality observe that either $g_{n+1}^{2}=f_{n+1}^{2}$ or $g_{n+1}^{2}<f_{n+1}^{2}$. In the first case, $2 g_{n+1}^{2}=2 f_{n+1}^{2} \geqq f_{n}^{2} \geqq g_{n}^{2}$, while in the second case $g_{n+1}^{2}-g_{n+2}^{2}=g_{n}^{2}$ $-g_{n+1}^{2}$ by the definition of $g^{2}$ and so $2 g_{n+1}^{2}-g_{n}^{2}=g_{n+2}^{2} \geqq 0$. Thus we have that $g_{n}^{2} \leqq 2 g_{n+1}^{2}$ for all values of $n$.

The hypothesis of the theorem requires that (1.1) not hold for $\psi$. By $A, \psi=\sigma$ and by monotonicity of $\sigma$ it is easy to see that $(1.1)$ is the same as

$$
\infty=\sum_{n=1}^{\infty} \frac{\sigma\left(2^{-n}\right)}{\sqrt{ } n}=\sum_{n=1}^{\infty} \frac{f_{n}}{\sqrt{ } n}
$$

We claim next that $\sum g_{n} / \sqrt{ } n=\infty$ also. To see this let $r$ and $s$, defined as above, 
be any two consecutive points where $f$ and $g$ are equal. Then if $r \leqq n \leqq(r+s) / 2$ we have by linearity

$$
\begin{aligned}
g_{n}^{2}=g_{r}^{2}+\left(g_{s}^{2}-g_{r}^{2}\right)(n-r) /(s-r) & \geqq g_{r}^{2}-g_{r}^{2}(n-r) /(s-r)=g_{r}^{2}(s-n) /(s-r) \\
& =f_{r}^{2}(s-n) /(s-r) \geqq f_{n}^{2}(s-n) /(s-r) \geqq f_{n}^{2} / 2
\end{aligned}
$$

and since $f_{n} / \sqrt{ } n$ decreases,

$$
\sum_{r \leqq n<s} \frac{f_{n}}{\sqrt{ } n} \leqq 2 \sum_{r \leqq n \leqq(r+s) / 2} \frac{f_{n}}{\sqrt{ } n} \leqq 2 \sqrt{ } 2 \sum_{r \leqq n \leqq(r+s) / 2} \frac{g_{n}}{\sqrt{ } n} \leqq 2 \sqrt{ } 2 \sum_{r \leqq n<s} \frac{g_{n}}{\sqrt{ } n}
$$

Now we define $a_{n}^{2}=g_{n}^{2}-g_{n+1}^{2}$. Since $g_{n}^{2}$ is convex, $a_{n}$ decreases monotonically. We now use the following inequality of Boas, proved in the appendix.

Lemma. Let $g_{n}^{2}=\sum_{j=n}^{\infty} a_{j}^{2}$ where $a_{j}$ decreases. Then

$$
\sum_{n=1}^{\infty} \frac{g_{n}}{\sqrt{ } n} \leqq 2 \sum_{n=1}^{\infty} a_{n}
$$

It follows from the lemma that (3.9) holds and we need only verify (3.3). Fix $t<1$ and let $N$ be the largest integer for which $2^{N} t \leqq 1$. By (3.8) we see that

$$
E(Y(s+t)-Y(s))^{2} \leqq \sum_{n=1}^{N} a_{n}^{2} 2^{2 n} t^{2}+4 \sum_{N+1}^{\infty} a_{n}^{2} .
$$

The second term on the right in (3.12) is $4 g_{N+1}^{2} \leqq 4 f_{N+1}^{2}=4 \sigma^{2}\left(2^{-N-1}\right) \leqq 4 \sigma^{2}(t)$ since $\sigma$ is monotonic by $A$. The first term on the right of (3.12) is less than

$$
\begin{aligned}
t^{2} \sum_{n=1}^{N} g_{n}^{2} 2^{2 n} & =t^{2} g_{N}^{2} \sum_{j=1}^{N} 2^{2 j}+t^{2} \sum_{n=1}^{N-1}\left(g_{n}^{2}-g_{n+1}^{2}\right) \sum_{j=1}^{n} 2^{2 j} \\
& \leqq \frac{1}{2} t^{2} g_{N}^{2} 2^{2 N \frac{4}{3}}+\frac{1}{2} t^{2} \sum_{n=1}^{N} g_{n}^{2} 2^{2 n \frac{4}{3}}
\end{aligned}
$$

since $g_{n}^{2}-g_{n+1}^{2} \leqq g_{n}^{2} / 2$ as we proved above, and since $\sum_{j=1}^{n} 2^{2 j} \leqq 2^{2 n}(4 / 3)$. From (3.13) we get upon subtracting the last term that

$$
t^{2} \sum_{n=1}^{N} g_{n}^{2} 2^{2 n} \leqq 2 t^{2} g_{N}^{2} 2^{2 N} \leqq 2 g_{N}^{2} .
$$

As before, $2 g_{N}^{2} \leqq 4 g_{N+1}^{2}<4 f_{N+1}^{2}=4 \sigma^{2}\left(2^{-N-1}\right) \leqq 4 \sigma^{2}(t)$ and so each term in (3.12) $\leqq 4 \sigma^{2}(t)$ and (3.3) holds with $K=8$. This proves the theorem in the case when $\sigma$ is monotonic and $\sigma^{2}\left(2^{-n}\right) \leqq 2 \sigma^{2}\left(2^{-n-1}\right)$. We point out that the proof would work as well even if $\sigma^{2}\left(2^{-n}\right) \leqq \alpha \sigma^{2}\left(2^{-n-1}\right)$ as long as $\alpha<4$. The case $\alpha=4$ is the general case because for any $\sigma$ and $h$ we have

$$
\sigma^{2}(2 h)=2 \int(1-\cos 2 h \lambda) d F(\lambda)=2 \int\left(2-2 \cos ^{2} h \lambda\right) d F(\lambda) \leqq 4 \sigma^{2}(h) .
$$


We now give another proof of the theorem, slightly more involved, but which works in general. We start from scratch at the expense of some repetition. Since the incremental variance $\sigma^{2}(t)$ has a least positive zero there is an $\varepsilon>0$ for which

$$
0<\sigma(\varepsilon / 2)=\min [\sigma(u): \varepsilon / 2 \leqq u \leqq \varepsilon],
$$

that is, the minimum of $\sigma$ on $[\varepsilon / 2, \varepsilon]$ is taken at the left endpoint. To see that such an $\varepsilon$ exists, let $2 z$ be the least positive zero of $\sigma$, and let $m(u)=\min [\sigma(x): u \leqq x \leqq z]$. We now choose $\varepsilon$ as the largest number $\leqq z$ for which $\sigma(\varepsilon / 2)=m(z / 2)$, which exists because $0<m(z / 2) \leqq \sigma(z / 2)$ and $\sigma$ tends to zero at zero. Then $0<\sigma(\varepsilon / 2) \leqq \sigma(x)$ for $\varepsilon / 2<x \leqq z / 2$ since $\varepsilon$ was largest and $\sigma(\varepsilon / 2) \leqq \sigma(x)$ for $z / 2 \leqq x \leqq \varepsilon$ because $\sigma(\varepsilon / 2)$ $=m(z / 2)$ and $\varepsilon \leqq z$. Thus (3.16) holds for $\varepsilon$.

Having chosen $\varepsilon$, we next define

$$
\psi(h)=\min [\sigma(u): h \leqq u \leqq \varepsilon] .
$$

$\psi$ dominates any nondecreasing minorant $\psi^{\prime}$ of $\sigma$ on $[0, \varepsilon]$ and so if the hypothesis of the theorem holds then (1.1) fails to hold for $\psi$, since

$$
\psi^{\prime}(h)=\min \left[\psi^{\prime}(u): h \leqq u \leqq \varepsilon\right] \leqq \min [\sigma(u): h \leqq u \leqq \varepsilon]=\psi(h)
$$

for $0 \leqq h \leqq \varepsilon$. Fixing $\psi$ as in (3.17) we have that if $2 t \leqq \varepsilon$,

$$
\begin{aligned}
\psi^{2}(2 t) & =\min \left[\sigma^{2}(2 u): t \leqq u \leqq \varepsilon / 2\right] \\
& =4 \min \left[\sigma^{2}(u): t \leqq u \leqq \varepsilon\right] \leqq 4 \min \left[\sigma^{2}(u): t \leqq u \leqq \varepsilon / 2\right]
\end{aligned}
$$

by (3.15) and so by (3.16) we have

$$
\psi^{2}(2 t) \leqq 4 \psi^{2}(t)
$$

Let now $f_{n}^{2}=\psi^{2}\left(2^{-n} \varepsilon\right), n=1,2, \ldots$, so that $f_{n}^{2} \downarrow$ and let $g_{n}^{2}$ be the largest convex minorant of $f_{n}^{2}$ defined as before. By hypothesis, $\int^{\infty} \psi\left(2^{-u} \varepsilon\right) u^{-1 / 2} d u=\infty$ and so $\sum f_{n} / \sqrt{ } n=\infty$ since $\psi$ is monotonic. By (3.11) we see that $\sum g_{n} / \sqrt{ } n=\infty$ as well.

We next define a convex subminorant $h^{2}$ of $g^{2}$ by extending certain edges of the graph of $g^{2}$, viewed as a convex polygon. Let $s_{0}=0$, and define $0<r_{1}<s_{1}<r_{2}$ $<s_{2}<\cdots$ as follows. Suppose that $s_{j-1}$ has been defined. Let $r_{j}$ be the first $n>s_{j-1}$ for which $g_{n}^{2}>2 g_{n+1}^{2}$ if there are such $n$. (Let $r_{j}=\infty$ if $g_{n}^{2} \leqq 2 g_{n+1}^{2}$ for all $n>s_{j-1}$.) If $r_{j}<\infty$, let $s_{j}$ be the first $n>r_{j}$ for which $g_{n}^{2} \leqq 2 g_{n+1}^{2}$, noting that $s_{j}<\infty$ since otherwise $g_{n}$ decreases exponentially contradicting $\sum g_{n} / \sqrt{ } n=\infty$. Now define $h_{n}=g_{n}$ for $s_{j-1} \leqq n \leqq r_{j}+1$ and for $r_{j}+1<n<s_{j}$ define $h$ so that $h_{n}^{2}-h_{n+1}^{2}=h^{2}\left(s_{j}\right)-h^{2}\left(s_{j}+1\right)$ $=g^{2}\left(s_{j}\right)-g^{2}\left(s_{j}+1\right)$ (where $h(n)$ is written for $h_{n}$ to avoid double subscripts). If $r_{j}=\infty$, set $h_{n}=g_{n}$ for all $n>s_{j-1}$. Since $h^{2}$ has been defined in $r_{j}+1<n<s_{j}$ by extending the edge between $s_{j}$ and $s_{j}+1$ of the graph of $g^{2}$ backwards throughout $r_{j}+1<n<s_{j}$, and since $g^{2}$ is convex, we must have $h_{n}^{2} \leqq g_{n}^{2}$ for all $n$ and we see that $h^{2}$ is also convex. We next show that $\sum h_{n} / \sqrt{ } n=\infty$. For $r_{j} \leqq n<s_{j}, g_{n+1}<g_{n} / \sqrt{ } 2$ and so $g_{n} \leqq g\left(r_{j}\right) \sqrt{ } 2^{r_{j}-n}$ for $r_{j} \leqq n<s_{j}$. Hence with $r=r_{j}, s=s_{j}$, we have

$$
\sum_{n=r}^{s-1} g_{n} / \sqrt{ } n \leqq(g(r) / \sqrt{ } r) \sum_{n=r}^{\infty} \sqrt{ } 2^{r-n} \leqq 4 g(r) / \sqrt{ } r=4 h(r) / \sqrt{ } r \leqq 4 \sum_{n=r}^{s-1} h(n) / \sqrt{ } n .
$$


Since $h_{n}=g_{n}$ for $s \leqq n<r_{j+1}$, it follows that $\sum g_{n} / \sqrt{ } n \leqq 4 \sum h_{n} / \sqrt{ } n$ and so $\sum h_{n} / \sqrt{ } n=\infty$.

Set $a_{n}^{2}=h_{n}^{2}-h_{n+1}^{2}$ in (3.6). Since $a_{n}$ decreases (3.9) holds by Boas' lemma. By Szidon's lemma the process $Y$ of (3.6) satisfies (3.4). By Slepian's lemma the theorem follows from (3.3) and (3.4) and the only thing left to show is that the right side of (3.12) is bounded by $K \sigma^{2}(t)$ for $t<\varepsilon$, where $N$ is the largest integer for which $2^{N} t \leqq \varepsilon, \varepsilon$ as above. The second sum in (3.12) is $4 h_{N+1}^{2} \leqq 4 g_{N+1}^{2} \leqq 4 f_{N+1}^{2}=4 \psi^{2}\left(2^{-N-1} \varepsilon\right)$ $\leqq 4 \psi^{2}(t) \leqq 4 \sigma^{2}(t)$. The first sum in (3.12) will be bounded by $K \sigma^{2}(t)$ if we can show that

$$
\sum_{n=1}^{N}\left(h_{n}^{2}-h_{n+1}^{2}\right) 2^{2 n} t^{2} \leqq K g_{N}^{2},
$$

because $g_{N}^{2} \leqq f_{N}^{2}=\psi^{2}\left(2^{-N} \varepsilon\right) \leqq 4 \psi^{2}\left(2^{-N-1} \varepsilon\right) \leqq 4 \psi^{2}(t) \leqq 4 \sigma^{2}(t)$, where we have used (3.18). It is the construction of $h$ that plays the crucial role in proving (3.19) and (3.19) is not necessarily true with the right side replaced by $K h_{N}^{2}$.

We begin with the observation that

$$
4 g_{n+1}^{2} \geqq g_{n}^{2} \text { for all } n=1,2, \ldots
$$

To prove (3.20) observe that either $g_{n+1}^{2}=f_{n+1}^{2}$ or $g_{n+1}^{2}<f_{n+1}^{2}$. In the first case, $4 g_{n+1}^{2}=4 f_{n+1}^{2} \geqq f_{n}^{2} \geqq g_{n}^{2}$ while in the second case, $g_{n+1}^{2}-g_{n+2}^{2}=g_{n}^{2}-g_{n+1}^{2}$ by the definition of $g^{2}$ and so $4 g_{n+1}^{2} \geqq 2 g_{n+1}^{2}=g_{n}^{2}+g_{n+2}^{2} \geqq g_{n}^{2}$. Thus (3.20) holds.

Now suppose that $N$ in (3.19) satisfies $s_{k} \leqq N<s_{k+1}$. For $j<k$ we have with $s=s_{j-1}, r=r_{j}, t=s_{j}$,

$$
\sum_{n=s+1}^{r}\left(h_{n}^{2}-h_{n+1}^{2}\right) 2^{2 n}=\sum_{n=s+1}^{r}\left(g_{n}^{2}-g_{n+1}^{2}\right) 2^{2 n} \leqq \sum_{n=s+1}^{r} g_{n}^{222 n}
$$

But summing by parts we get

$$
\begin{aligned}
\sum_{n=s+1}^{r} g_{n}^{2} 2^{2 n} & =g_{r}^{2} \sum_{n=s+1}^{r} 2^{2 k}+\sum_{n=s+1}^{r-1}\left(g_{n}^{2}-g_{n+1}^{2}\right) \sum_{k=s+1}^{n} 2^{2 k} \\
& \leqq \frac{4}{3} g_{r}^{2} 2^{2 r}+\frac{4}{3} \sum_{n=s+1}^{r-1}\left(g_{n}^{2}-g_{n+1}^{2}\right) 2^{2 n}
\end{aligned}
$$

Since $g_{n}^{2} \leqq 2 g_{n+1}^{2}$ for $s<n \leqq r-1, g_{n}^{2}-g_{n+1}^{2} \leqq g_{n}^{2} / 2$ for $s+1 \leqq n \leqq r-1$ and substituting in (3.22) we get

$$
\sum_{n=s+1}^{r} g_{n}^{22} 2^{2 n} \leqq \frac{2}{3} g_{r}^{2} 2^{2 r}+\frac{2}{3} \sum_{n=s+1}^{r} g_{n}^{222^{2 n}}
$$

Subtracting the last term in (3.23) from both sides and using (3.21) gives

$$
\sum_{n=s+1}^{r}\left(h_{n}^{2}-h_{n+1}^{2}\right) 2^{2 n} \leqq 2 g_{r}^{222^{2 r}}
$$


Since $h$ is linear in the range $r+1<n \leqq t+1$ we have

$$
\begin{aligned}
\sum_{n=r+2}^{t}\left(h_{n}^{2}-h_{n+1}^{2}\right) 2^{2 n} & =\left(h_{t}^{2}-h_{t+1}^{2}\right) \sum_{n=r+2}^{t} 2^{2 n} \\
& \leqq\left(g_{t}^{2}-g_{t+1}^{2}\right) 2^{2 t \frac{4}{3}} \leqq 2 g_{t}^{2} 2^{2 t}
\end{aligned}
$$

Since $\left(h_{r+1}^{2}-h_{r+2}^{2}\right) 2^{2(r+1)} \leqq 4 h_{r+1}^{2} 2^{2 r} \leqq 4 h_{r}^{2} 2^{2 r}=4 g_{r}^{2} 2^{2 r}$ we have from (3.24) and (3.25) that

$$
\begin{aligned}
\sum_{n=s_{j-1}+1}^{s_{j}}\left(h_{n}^{2}-h_{n+1}^{2}\right) 2^{2 n} & \leqq 6 g\left(r_{j}\right)^{2} 2^{2 r_{j}}+2 g^{2}\left(s_{j}\right) 2^{2 s_{j}} \\
& \leqq 8 g^{2}\left(s_{j}\right) 2^{2 s_{j}}
\end{aligned}
$$

the last inequality being valid because by (3.20) $g_{n}^{2} \leqq 4 g_{n+1}^{2}$ for all $n$ and so $g(n)^{2} 2^{2 n}$ is monotonically increasing in $n$. Now again by (3.20), $g_{n}^{2} \leqq 4 g_{n+1}^{2}$ for $s_{j-1} \leqq n<s_{j}$ but there is at least one value of $n$ in the range $s_{j-1} \leqq n<s_{j}$ for which $g_{n}^{2} \leqq 2 g_{n+1}^{2}$, namely $n=s_{j-1}$. Hence

$$
g\left(s_{j-1}\right)^{2} 2^{2 s_{j-1}} \leqq \frac{1}{2} g\left(s_{j}\right)^{2} 2^{2 s_{j}} .
$$

Summing the inequalities (3.26) and using (3.27) we get

$$
\sum_{n=1}^{s_{k}}\left(h_{n}^{2}-h_{n+1}^{2}\right) 2^{2 n} \leqq 8 g\left(s_{k}^{2}\right) 2^{2 s_{k}}\left(1+\frac{1}{2}+\frac{1}{4}+\cdots+\frac{1}{2^{k}}\right) .
$$

The same proof as in (3.24) shows that if $s_{k} \leqq N \leqq r_{k}$ that

$$
\sum_{n=s_{k}+1}^{N}\left(h_{n}^{2}-h_{n+1}^{2}\right) 2^{2 n} \leqq 2 g_{N}^{2} 2^{2 N}
$$

and the same proof as in (3.25) shows that (3.29) is valid also for $r_{k}<N<s_{k+1}$. Thus we have from (3.28) and (3.29)

$$
\sum_{n=1}^{N}\left(h_{n}^{2}-h_{n+1}^{2}\right) 2^{2 n} \leqq 18 g_{N}^{2} 2^{2 N}
$$

again using the monotonicity of $g_{n}^{22^{2 n}}$. Since $t^{2} 2^{2 N} \leqq \varepsilon^{2}$ we see that (3.19) is valid with $K=18 \varepsilon^{2}$. The theorem is proved.

4. Discontinuous processes which are incrementally continuous but not sequentially continuous. A process $X$ will be called incrementally continuous if for every monotonic (either increasing or decreasing) bounded sequence $t=t_{1}, t_{2}, \ldots$,

$$
X\left(t_{n+1}\right)-X\left(t_{n}\right) \rightarrow 0 \text { as } n \rightarrow \infty
$$

except for a set $A(t)$ of paths $X$ with $P(A(t))=0$. Of course if $X$ consists of a single function then that function must be continuous, but the Poisson process is an example of a discontinuous process which is incrementally continuous. On the other hand, it seems to be difficult to find a process which is incrementally 
continuous without also being sequentially continuous, that is whenever $t_{n} \rightarrow t$, $X\left(t_{n}\right) \rightarrow$ limit, except for a null set $A(t)$. Since it can be shown $\left({ }^{2}\right)$ that a sequentially continuous Gaussian process always has continuous sample paths, examples of processes with the desired properties can be constructed by using the theorem of $\$ 3$ and the following theorem.

THEOREM. If $X$ has covariance $\rho$ of Polya's type then $X$ is incrementally continuous if and only if (1.4) holds.

Proof. The property of Polya-type processes that we make use of is that if $s_{1}<s_{2}<t_{1}<t_{2}$, then

$$
E\left(X\left(s_{2}\right)-X\left(s_{1}\right)\right)\left(X\left(t_{2}\right)-X\left(t_{1}\right)\right) \leqq 0 .
$$

Indeed, if $t_{2}-s_{2}<t_{1}-s_{1}$ we write the left side of (4.2) as

$$
\begin{aligned}
\left(\rho\left(t_{2}-s_{2}\right)-\rho\left(t_{1}-s_{2}\right)\right)-\left(\rho\left(t_{2}-s_{1}\right)-\rho\left(t_{1}-s_{1}\right)\right) & =\left(\rho^{\prime}\left(\theta_{2}\right)-\rho^{\prime}\left(\theta_{1}\right)\right)\left(t_{2}-t_{1}\right) \\
& =\rho^{\prime \prime}\left(\theta_{3}\right)\left(\theta_{2}-\theta_{1}\right)\left(t_{2}-t_{1}\right) \leqq 0
\end{aligned}
$$

since $0<\theta_{2}<t_{2}-s_{2}<t_{1}-s_{1}<\theta_{1}$. On the other hand if $t_{1}-s_{1}<t_{2}-s_{2}$ we write the left side of (4.2) as $\left(\rho\left(t_{1}-s_{1}\right)-\rho\left(t_{1}-s_{2}\right)\right)-\left(\rho\left(t_{2}-s_{1}\right)-\rho\left(t_{2}-s_{2}\right)\right)$ and proceed analogously. Thus (4.2) holds.

Now we use two lemmas from [7], omitting their proofs.

Lemma 1. Let $X$ and $Y$ be Gaussian variables with zero mean and suppose $E X Y \leqq 0$. Then if $a \geqq 0, b \geqq 0$,

$$
P(X \geqq a, Y \geqq b) \leqq P(X \geqq a) P(Y \geqq b) .
$$

LEMMA 2. Let $B_{1}, B_{2}, \ldots$ be events with $P\left(B_{j} B_{k}\right) \leqq P\left(B_{j}\right) P\left(B_{k}\right)$ for all $j \neq k$. If $\sum P\left(B_{n}\right)=\infty$ then $P\left(B_{n}\right.$ infinitely often $)=1$.

Let $\boldsymbol{t}$ be a monotone sequence and $\varepsilon>0$. Define

$$
B_{n}=\left\{X\left(t_{n+1}\right)-X\left(t_{n}\right) \geqq \varepsilon\right\}, \quad n=1,2, \ldots
$$

By (4.2), Lemmas 1 and 2, and the usual Borel-Cantelli lemma, we see that $X$ is incrementally continuous if and only if $\sum P\left(B_{n}\right)<\infty$ for every monotonic sequence $t$. Supposing that $t_{n} \uparrow t$ which involves no loss of generality, we see that $\delta_{n}=t_{n+1}$ $-t_{n} \geqq 0$ must satisfy $\sum \delta_{n}<\infty$. We have

$$
P\left(B_{n}\right)=P\left\{\eta \geqq \varepsilon / \sigma\left(\delta_{n}\right)\right\}
$$

where $\eta$ is a standard normal variable. If (1.4) fails to hold then there exist $u_{n} \downarrow 0$ and $\theta>0$ for which $\sigma^{2}\left(u_{n}\right)>\theta / \log \left(1 / u_{n}\right)$. If we let $\left(\delta_{1}, \delta_{2}, \ldots\right)=\left(u_{1}, u_{1}, \ldots, u_{1}\right.$, $\left.u_{2}, u_{2}, \ldots, u_{2}, u_{3}, \ldots\right)$ where the block of $u_{1}$ 's is of length $A_{1} \geqq 0$, the block of $u_{2}$ 's

(2) See Appendix 4. 
is of length $A_{2} \geqq 0$, and so on, we can make $\sum \delta_{n}<\infty$ and $\sum P\left(B_{n}\right)=\infty$ provided that we can choose the $A$ 's so that

$$
\sum A_{n} u_{n}<\infty
$$

and

$$
\sum A_{n} \exp \left(-\varepsilon^{2} / \sigma^{2}\left(u_{n}\right)\right)=\infty
$$

hold. The sum in (4.6) is at least $\sum A_{n} u_{n}^{\varepsilon^{2} / \theta}$. If we choose $\varepsilon^{2}=\theta / 2$, it is clear that there can be found nonnegative integers $A_{1}, A_{2}, \ldots$, for which $\sum A_{n} u_{n}<\infty$ and $\sum A_{n} u_{n}^{1 / 2}$ $=\infty$. Thus if (1.4) fails for $X$ of Polya type then $X$ is not incrementally continuous.

On the other hand, if (1.4) holds, then $X$ is incrementally continuous even if $X$ is not of Polya's type because $\left(\varepsilon^{2} / 2 \sigma^{2}\left(\delta_{n}\right)\right)^{-1}=o\left(1 / \log \left(1 / \delta_{n}\right)\right)$ and it follows that $\sum P\left(B_{n}\right)<\infty$ since $P\left(B_{n}\right) \leqq \delta_{n}$ for sufficiently large $n$.

It is easy to find covariances which satisfy (1.4) but not (1.1) and thereby construct incrementally continuous processes which are not sequentially continuous.

5. Examples. We give first a continuous process with $\lim \sup (\log 1 / h) \sigma^{2}(h) \neq 0$ thereby showing that the condition that $X$ be of Polya type cannot be dropped entirely in proving that $X$ is not incrementally continuous in $\$ 4$. At the same time, $\sigma$ of the example also fails to satisfy (1.2) and thereby shows that (1.2) cannot be necessary for sample continuity in general. The example is

$$
X(t)=\sum_{1}^{\infty} \frac{1}{n^{2}}\left(\eta_{n} \cos 2^{2^{n}} t+\eta_{n}^{\prime} \sin 2^{2^{n}} t\right)
$$

where $\eta$ and $\eta^{\prime}$ are independent standard normal sequences. $X$ is continuous because $\sum\left(\left|\eta_{n}\right|+\left|\eta_{n}^{\prime}\right|\right) / n^{2}<\infty$, a.s. On the other hand, we have

$$
\left(\log 2^{2^{k}}\right) \sigma^{2}\left(1 / 2^{2^{k}}\right) \geqq(1-\cos 1)(\log 2) 2^{k} / k^{4} \rightarrow \infty
$$

and so $\lim \sup (\log 1 / h) \sigma^{2}(h) \neq 0$. A short calculation shows that (1.2) fails to hold. Of course, $\sigma^{2}$ is not monotonic.

We remark that an extension of the above example shows that there is no simple analogue of Slepian's lemma in terms of spectral distribution functions (s.d.f.). In fact if $F$ is the s.d.f. of any discontinuous process then there is a s.d.f. $G$ of a continuous process $Y$ with $G(x) \leqq F(x)$ for all $x \geqq 0, G(\infty)=F(\infty)$. Indeed this is trivially seen by taking $Y(t)=\sum a_{n}\left(\eta_{n} \cos \theta_{n} t+\eta_{n}^{\prime} \sin \theta_{n} t\right)$ where $\eta$ and $\eta^{\prime}$ are as in (3.6). If $\sum\left|a_{n}\right|<\infty, Y$ is continuous no matter what values the $\theta$ 's take and if $\theta_{n} \rightarrow \infty$ sufficiently fast, it is clear that $G$ will have uniformly fatter tails than $F$. Similarly, it is shown that if $F$ is any s.d.f. with $F \not \equiv 0$ then $G_{1}$ and $G_{2}$ may be found with $G_{1} \leqq F \leqq G_{2}$ and $G_{1}(\infty)=G_{2}(\infty)$ where the corresponding processes are either continuous or discontinuous.

The next example shows that the condition

$$
\sum s_{n}^{1 / 2}<\infty, \quad s_{n}=F\left(2^{n+1}\right)-F\left(2^{n}\right),
$$


where $F$ is the spectral cumulative distribution function of $X$, fails to be sufficient for continuity of $X$ in general. Our example, which is of Polya type, is defined as follows:

Let $\mu$ be the measure having a point mass

$$
\lambda_{m}=\left(1 / 2^{m}\right)^{1 / 2} \text { at } p_{m}=1 / 2^{2^{m}}
$$

$m=1,2, \ldots$ Define $F(x)$ by setting $F^{\prime}(x)=f(x)$ for all $x$, where

$$
f(x)=\int_{0}^{1} \frac{1-\cos x t}{x^{2} t^{2}} t d \mu(t)
$$

If $p_{m}<x^{-1}<p_{m+1}$

$$
f(x) \leqq \int_{0}^{1 / x} t d \mu(t)+\int_{1 / x}^{1} \frac{2}{x^{2} t} d \mu(t) \leqq 2 \lambda_{m+1} p_{m+1}+4 x^{-2} \lambda_{m} p_{m}^{-1}
$$

and so, for $0 \leqq k<2^{m}$,

$$
F\left(2^{2^{m}+k+1}\right)-F\left(2^{2^{m}+k}\right)=S_{2^{2^{m}+k}} \leqq 2 \lambda_{m+1} p_{m+1} 2^{2^{m}+k}+4 \lambda_{m} p_{m}^{-1} 2^{-2^{m-k}} .
$$

It follows easily that (5.3) holds. To prove that $X$ is not continuous it suffices to check that (1.2) fails to hold because $X$ is of Polya type, as is seen by noting that

$$
\begin{aligned}
f(x) & =\int_{0}^{1} \rho(t) \cos x t d t=-\frac{1}{x} \int_{0}^{1} \rho^{\prime}(t) \sin x t d t \\
& =\int_{0}^{1} \frac{1-\cos x t}{x^{2} t^{2}} t^{2} d \rho^{\prime}(t)
\end{aligned}
$$

where we have set $t d \rho^{\prime}(t)=d \mu(t), \rho(1)=\rho^{\prime}(1)=0, \mu$ as in (5.4). We have

$$
\begin{aligned}
\rho(0)-\rho(t) & =\int_{0}^{t}-\rho^{\prime}(u) d u=-\rho^{\prime}(t) t+\int_{0}^{t} d \mu(u) \\
& =t \int_{t}^{1} \frac{1}{u} d \mu(u)+\int_{0}^{t} d \mu(u) \geqq \int_{0}^{t} d \mu(u) .
\end{aligned}
$$

Thus if $p_{m+1}<t<p_{m}, \rho(0)-\rho(t) \geqq \lambda_{m+1}$ and it is a simple matter to check that (1.2) fails to hold. Thus $X$ is discontinuous in spite of (5.3). Of course, $s_{n}$ in this case is not a monotonic sequence.

\section{Appendix.}

1. Proof of Slepian's lemma. The intuitive explanation of Slepian's inequality is that although $X$ and $Z$ are instanteously of equal variance, $X$ oscillates more than $Z$ because it is less correlated. It is enough to prove (3.5) for finite sets $I=$ $\left\{t_{1}, \ldots, t_{n}\right\}$. The left side of (3.5) is then $1-Q$ where $Q=Q(r)$ is given by

$$
Q(\boldsymbol{r})=\int_{-\infty}^{M} d x_{1} \cdots \int_{-\infty}^{M} d x_{n} g\left(x_{1}, \ldots, x_{n} ; \boldsymbol{r}\right)
$$


and the Gaussian density $g(x, r)$ is given in terms of its characteristic function by

(A.2) $g(\boldsymbol{x}, \boldsymbol{r})=\int_{-\infty}^{\infty} d \xi_{1} \cdots \int_{-\infty}^{\infty} d \xi_{n}(2 \pi)^{-n} \exp \left(i \sum x_{j} \xi_{j}-(1 / 2) \sum \sum r_{j k} \xi_{j} \xi_{k}\right)$

and $r_{j k}=E X\left(t_{j}\right) X\left(t_{k}\right), r=\left\{r_{j k}\right\}$. From (A.2) we obtain immediately that $\partial g / \partial r_{j k}$ $=\partial^{2} g / \partial x_{j} \partial x_{k}$ for $j \neq k$ and so differentiating (A.1) with respect to $r_{12}$ say we get

$$
\frac{\partial}{\partial r_{12}} Q(\boldsymbol{r})=\int_{-\infty}^{M} d x_{1} \cdots \int_{-\infty}^{M} d x_{n} \frac{\partial^{2}}{\partial x_{1} \partial x_{2}} g\left(x_{1}, x_{2}, \ldots, x_{n}, \boldsymbol{r}\right) .
$$

Integrating we get

$$
\frac{\partial}{\partial r_{12}} Q(\boldsymbol{r})=\int_{-\infty}^{M} d x_{3} \cdots \int_{-\infty}^{M} d x_{n} g\left(M, M, x_{3}, \ldots, x_{n}, \boldsymbol{r}\right) .
$$

Thus $\partial Q(\boldsymbol{r}) / \partial r_{j k} \geqq 0$ for all $j \neq k$. Now suppose that $s_{j k}=E Z\left(t_{j}\right) Z\left(t_{k}\right)$ and let

$$
r_{j k}(\lambda)=\lambda r_{j k}+(1-\lambda) s_{j k} .
$$

Then $\boldsymbol{r}(\lambda)=\left\{\boldsymbol{r}_{j k}(\lambda)\right\}$ is positive definite and so $q(\lambda)=1-Q(\boldsymbol{r}(\lambda))$ is defined. We see that

$$
\begin{aligned}
q^{\prime}(\lambda) & =-\sum_{j} \sum_{k}\left(\partial / \partial r_{j k}\right) Q(r(\lambda)) \cdot d r_{j k}(\lambda) / d \lambda \\
& =-\sum_{j} \sum_{k}\left(\partial / \partial r_{j k}\right) Q(r(\lambda)) \cdot\left(r_{j k}-s_{j k}\right) \geqq 0
\end{aligned}
$$

since $r_{j j}=s_{j j}$ and for $j \neq k, \partial Q / \partial r_{j k} \geqq 0$ and $s_{j k} \geqq r_{j k}$. Thus

$$
q(1)=1-Q(r)=P\left\{\max X\left(t_{j}\right) \geqq M\right\} \geqq q(0)=1-Q(s)=P\left\{\max Z\left(t_{j}\right) \geqq M\right\},
$$

proving (3.5).

2. Proof of Boas' inequality. The following proof was shown to us by $\mathbf{H}$. $\mathrm{O}$. Pollak. We have since $a_{1} \geqq a_{2} \geqq \cdots$, for each $m \geqq n$

$$
\begin{aligned}
\left(\sum_{k=n}^{m} a_{k}^{2}\right)^{1 / 2}-\left(\sum_{k=n}^{m-1} a_{k}^{2}\right)^{1 / 2} & =a_{m}^{2}\left(\left(\sum_{k=n}^{m} a_{k}^{2}\right)^{1 / 2}+\left(\sum_{k=n}^{m-1} a_{k}^{2}\right)^{1 / 2}\right)^{-1} \\
& \leqq a_{m}^{2} /(m+1-n)^{1 / 2} a_{m}=a_{m} /(m+1-n)^{1 / 2}
\end{aligned}
$$

Adding on $m$, we get

$$
\begin{aligned}
\sum_{m=n}^{\infty}\left(\left(\sum_{k=n}^{m} a_{k}^{2}\right)^{1 / 2}-\left(\sum_{k=n}^{m-1} a_{k}^{2}\right)^{1 / 2}\right) & =\left(\sum_{k=n}^{\infty} a_{k}^{2}\right)^{1 / 2}=g_{n} \\
& \leqq \sum_{m=n}^{\infty} a_{m} /(m+1-n)^{1 / 2}
\end{aligned}
$$


Dividing by $\sqrt{ } \boldsymbol{n}$ and adding, we get

$$
\begin{aligned}
\sum_{n=1}^{\infty} g_{n} / \sqrt{ } n & \leqq \sum_{n=1}^{\infty}(1 / \sqrt{ } n) \sum_{m=n}^{\infty} a_{m} /(m+1-n)^{1 / 2} \\
& =\sum_{m=1}^{\infty} a_{m} \sum_{n=1}^{m} 1 /(n(m+1-n))^{1 / 2} \\
& \leqq 2 \sum_{m=1}^{\infty} a_{m},
\end{aligned}
$$

proving the lemma.

We remark finally that the reverse inequality, $\sum a_{n} \leqq 2 \sum g_{n} / \sqrt{ } n$ is valid even without assuming that the $a$ 's are monotonic. Indeed, we may write

$$
\sum_{n=1}^{\infty} a_{n}=\sum_{n=1}^{\infty} \sum_{m=n}^{\infty} \frac{1}{m} a_{m} \leqq \sum_{n=1}^{\infty} g_{n}\left(\sum_{m=n}^{\infty} 1 / m^{2}\right)^{1 / 2}
$$

and the remark follows.

3. Footnote $1, \S 2$. Actually Belyaev's theorem shows only that a discontinuous Gaussian process is a.s. unbounded on any interval $I$. To see that $X$ is in fact a.s. unbounded on any set $S$ dense in $I$, we proceed as follows. If $S^{\prime} \subset I$ is a countable set which acts as a set of separability for $X$, then, since $X$ is unbounded on $I$, $\sup \left[|X(t)|: t \in S U S^{\prime}\right]=\infty$, a.s. But $\sup [|X(t)|: t \in S]=\sup \left[|X(t)|: t \in S U S^{\prime}\right]$ w.p.l. because every point $t \in S^{\prime}$ is a limit of points $t_{n} \in S$ and so $X\left(t_{n^{\prime}}\right) \rightarrow X(t)$ a.s. along some subsequence $\left\{t_{n^{\prime}}\right\}$ of $\left\{t_{n}\right\}$ since $X\left(t_{n}\right) \rightarrow X(t)$ in the mean. Since $S^{\prime}$ is countable we see that $\sup [|X(t)|: t \in S]=\sup \left[|X(t)|: t \in S U S^{\prime}\right]=\infty$ w.p.l. Thus the claim that (2.3) is sufficient for the theorem is proved.

4. Footnote $2, \$ 4$. To see that a discontinuous Gaussian process is not sequentially continuous, note first that as a result of Appendix 3 above if $X$ is discontinuous and $a<b$,

$$
P(|X(a+(b-a) j \mid n)| \leqq M, j=0,1, \ldots, n) \rightarrow 0, \quad \text { as } n \rightarrow \infty .
$$

Define $t_{2}=t_{4}=\cdots=0$ and choose $0<a_{j}<b_{j}, j=1,2, \ldots$, with $b_{j} \downarrow 0$. Define $t_{1}, t_{3}, \ldots$, and $n(0)=0<n(1)<\cdots$ inductively as follows: Choose for $j=1,2, \ldots$, $t_{2 n(j-1)+1}, t_{2 n(j-1)+3}, \ldots, t_{2 n(j)-1}$ so each belong to $\left(a_{j}, b_{j}\right)$ and together satisfy for each $j=1,2, \ldots$

$$
P\left(\left|A\left(t_{2 i+1}\right)\right| \leqq j, n(j-1) \leqq i<n(j)\right)<1 / j,
$$

which can be done on account of (D.1). We see that $t_{n} \rightarrow 0$ but $X\left(t_{n}\right)$ do not have a limit because $P\left(X\left(t_{2 n+1}\right)-X\left(t_{2 n}\right) \rightarrow 0\right)=P\left(X\left(t_{2 n+1}\right)-X(0) \rightarrow 0\right) \leqq P\left(\left|X\left(t_{2 n+1}\right)\right|\right.$, $n=1,2, \ldots$ is bounded) $=0$ by (D.2). Thus $X$ is not sequentially continuous.

\section{REFERENCES}

1. Yu. K. Belyaev, Continuity and Hölder's conditions for sample functions of stationary Gaussian processes, Proc. Fourth Berkeley Sympos. Math. Statist. and Prob. (Berkeley, Calif., 1960), vol. 2, Univ. of California Press, Berkeley, 1961, pp. 23-33. MR 26 \#815. 
2. R. P. Boas, Jr. Inequalities for monotonic series, J. Math. Anal. Appl. 1 (1960), 121-126. MR 22 \#8244.

3. R. L. Dobrushin, Properties of sample functions of stationary Gaussian processes, Teor. Veroyatnost. i Primenen. 5 (1960), 132-134. MR 25 \#2644.

4. D. M. Eaves, Sample functions of Gaussian random homogeneous fields are either continuous or very irregular, Amer. Math. Statist. 38 (1967), 1579-1582. MR 36 \#5998.

5. X. Fernique, Continuité des processus Gaussiens, C. R. Acad. Sci. Paris 258 (1964), 6058-6060. MR 29 \#1662.

6. G. A. Hunt, Random Fourier transforms, Trans. Amer. Math. Soc. 71 (1951), 38-69. MR 14, 465.

7. M. B. Marcus, Hölder conditions for Gaussian processes with stationary increments, Trans. Amer. Math. Soc. 134 (1968), 29-52. MR 37 \#5930.

8. M. Nisio, On the extreme values of Gaussian processes, Osaka J. Math. 4 (1967), 313-326. MR 37 \#2309.

9. - On the continuity of stationary Gaussian processes, Nagoya Math. J. 34 (1969), 89-104. MR 39 \#2210.

10. H. J. Landau and L. A. Shepp, On the supremum of a Gaussian process, Sankhya (to appear).

11. D. Slepian, The one-sided barrier problem for Gaussian noise, Bell System Tech. J. 41 (1962), 463-501. MR 24 \#A3017.

12. A. Zygmund, Trigonometric series, Monografie Mat., Bd. 5, PWN, Warsaw, 1935, 2nd ed., Cambridge Univ. Press, New York, 1952. MR 21 \#6498.

13. R. M. Dudley, The sizes of compact subsets of Hilbert space and continuity of Gaussian processes, J. Functional Analysis 1 (1967), 290-330. MR 36 \#3405.

14. A. M. Garsia, E. Rodemich and H. Rumsey Jr., A real variable lemma and the continuity of paths of some Gaussian processes (to appear).

15. M. R. Leadbetter and E. W. Weissner, On continuity and other analytic properties of stochastic process sample functions, Proc. Amer. Math. Soc. 22 (1969), 291-294.

16. M. B. Marcus, $A$ bound for the distribution of the maximum of continuous Gaussian processes, Ann. Math. Statist. 41 (1970), 305-309.

NORTHWESTERN UNIVERSITY,

EVANSTON, IlLINOIS 60201

Bell Telephone Laboratories, Inc.,

MurRay Hill, New Jersey 07974 